\section{ORIGINAL RESEARCH}

\author{
J.C. Carter \\ D.C. Lanham \\ D. Pham \\ G. Bibat \\ S. Naidu \\ W.E. Kaufmann
}

\title{
Selective Cerebral Volume Reduction in Rett Syndrome: A Multiple-Approach MR Imaging Study
}

\begin{abstract}
BACKGROUND AND PURPOSE: Previous studies have examined volumetric abnormalities in Rett syndrome (RTT), using MR imaging and focusing on selective changes. However, these studies preceded the identification of MECP2 as the gene mutated in most RTT cases. We studied regional brain volume changes as noted by MR imaging in girls with RTT who had mutations in the MECP2 gene and more or less severe clinical outcomes to further characterize the neuroanatomy of RTT and its correlations with clinical severity.
\end{abstract}

\begin{abstract}
MATERIALS AND METHODS: Complementary semiautomated Talairach- and voxel-based approaches were used to study spoiled gradient-recalled acquisition sequence MR imaging scans from 23 girls with MECP2 mutations/RTT, including a pair of discordant monozygotic twins and 25 age-matched control girls. Both absolute and relative volumetric changes were examined to account for the well-documented global reduction in brain volume seen in RTT.
\end{abstract}

\begin{abstract}
RESULTS: Absolute volumetric reductions were observed throughout the brain in RTT. Selective/ relative decreases in parietal lobe gray matter, particularly in the dorsal parietal region, and mild, diffuse reductions in cortical white matter were observed in the RTT group compared with control subjects. In girls with RTT and a more severe phenotype, anterior frontal lobe volumes were relatively more reduced. Twin comparisons revealed selective preservation of the occipital cortex.
\end{abstract}

CONCLUSION: Selective reductions of dorsal parietal gray matter and preservation of the occipital cortex seem to be basic neuroanatomic features of RTT, whereas preferential reduction of the anterior frontal lobe appears to be a correlate of clinical severity in this disorder. The most affected brain regions include those that may underlie key functional deficits observed in RTT.

O riginally described by Andreas Rett in 1966, Rett syndrome (RTT) is a neurodevelopmental disorder predominantly affecting girls ${ }^{1-4}$ that is caused by a mutation in $M E C P 2$, a gene located on the long arm of the $\mathrm{X}$ chromosome (Xq28), which encodes the transcriptional repressor methylCpG-binding protein 2..$^{5-9}$ More than 200 pathogenic mutations have been identified, ${ }^{10}$ and evidence suggests that genotypic differences may account for clinical presentation. ${ }^{11,12}$ Before availability of genetic testing, the diagnosis of RTT was based solely on a collection of clinical features organized into age-related stages. ${ }^{13,14}$ Although girls meeting all of these clinical criteria are classified as having "classic" RTT, there are also variants of the disorder marked by a less severe clinical presentation, including preserved speech or hand use, normal head circumference, or delayed symptom onset. ${ }^{15}$ Given the evidence for an association between specific MECP2 mutations and clinical severity, ${ }^{11,16-18}$ as well as for considerable phenotypic variability between and within genotypes, ${ }^{12,19}$ clinical se-

Received July 20, 2007; accepted after revision September 6.

From the Center for Genetic Disorders of Cognition and Behavior (J.C.C., W.E.K.), Department of Developmental Cognitive Neurology (D.C.L.), and Neurogenetics Unit (G.B., S.N.), Kennedy Krieger Institute, Baltimore, Md; Departments of Radiology and Radiological Science (D.P., W.E.K.), Neurology (S.N., W.E.K.), Pediatrics (G.B., S.N., W.E.K.), Pathology (W.E.K.), and Psychiatry and Behavioral Sciences (W.E.K.), Johns Hopkins University School of Medicine, Baltimore, Md.

This work was supported by grants P01 HD 24448 and P30 HD 24061 from the National Institutes of Health.

Please address correspondence to Walter E. Kaufmann, Center for Genetic Disorders of Cognition and Behavior, Kennedy Krieger Institute, 3901 Greenspring Ave, Baltimore, MD 21211; e-mail: kaufmann@kennedykrieger.org

三E indicates article with supplemental on-line tables.

DOI 10.3174/ajnr.A0857 verity-based examinations of neuroanatomy in RTT represent an elucidative avenue of investigation.

Clinical, neuropathologic, and neuroimaging studies have shown that there is an early and marked decrease in brain and cerebral size in RTT. ${ }^{20}$ Morphologic correlates of these include generalized reductions in neuronal soma size and dendritic arborizations, primarily affecting the cerebral cortex. ${ }^{20}$ Early neuroimaging studies ${ }^{21,22}$ confirmed the approximately $25 \%$ reduction in total brain volume demonstrated in neuropathologic studies ${ }^{23}$ and also showed a preferential reduction of cortical volume, with relatively equal involvement of gray matter (GM) and white matter (WM). ${ }^{21}$ Studies of regional cortical involvement demonstrated reductions in the relative proportions of GM in the prefrontal, posterior frontal, and anterior temporal regions, with relative preservation in the posterior occipital region $^{22}$ and uniform reductions in WM volume throughout the brain. ${ }^{24}$ These analyses were based on proportions of specific tissue volumes to whole lobar volumes rather than absolute tissue volumes. Blood flow studies also suggest selective frontal lobe involvement. ${ }^{25,26}$ However, neuroimaging studies to date have relied on clinical phenotype-based parameters for the classification of RTT, without confirmation of a mutation in MECP2. This presents the potential problem of examining an etiologically inhomogeneous cohort, thereby complicating the delineation of a specific neuroanatomic phenotype.

In the present study, we sought to address the issue of preferential volumetric reductions in the RTT brain by using complementary atlas- (Talairach) and voxel-based MR imaging morphometric approaches. In the former, we examined both absolute and relative/selective changes in GM and WM vol- 


\begin{tabular}{|c|c|c|c|c|c|c|c|c|c|}
\hline \multirow[b]{2}{*}{ Variable } & \multirow[b]{2}{*}{$n$} & \multirow{2}{*}{$\begin{array}{c}\text { Age, } \\
\text { Mean } \pm S D \\
y\end{array}$} & \multirow{2}{*}{$\begin{array}{c}\text { Head } \\
\text { Circumference, } \\
\text { Mean } \pm \text { SD, } \\
\text { cm }\end{array}$} & \multicolumn{6}{|c|}{$\begin{array}{c}\text { Clinical Severity Scores, } \\
\text { Mean } \pm \text { SD }\end{array}$} \\
\hline & & & & Total & $\begin{array}{l}\text { Head } \\
\text { Growth }\end{array}$ & Seizures & $\begin{array}{l}\text { Respiratory } \\
\text { Irregularities }\end{array}$ & Scoliosis & $\begin{array}{c}\text { Gait } \\
\text { Abnormalities }\end{array}$ \\
\hline All RTT subjects & 22 & $8.6 \pm 1.8$ & $49.5 \pm 2.1$ & $7.8 \pm 2.4$ & $2.5 \pm 0.8$ & $1.2 \pm 1.0$ & $1.7 \pm 0.8$ & $0.7 \pm 1.0$ & $1.6 \pm 1.0$ \\
\hline Affected MZ twin & & 8.4 & 50.5 & 8 & 3 & 1 & 2 & 0 & 2 \\
\hline More severe (gait criterion) & 12 & $8.8 \pm 1.6$ & $49.3 \pm 2.4$ & $9.6 \pm 1.3^{*}$ & $2.6 \pm 0.7$ & $1.4 \pm 1.0$ & $1.8 \pm 0.8$ & $1.7 \pm 1.2^{*}$ & $2.4 \pm 0.7^{*}$ \\
\hline Less severe (gait criterion) & 10 & $8.3 \pm 2.1$ & $49.4 \pm 1.8$ & $5.9 \pm 1.5$ & $2.5 \pm 1.0$ & $1.0 \pm 1.1$ & $1.6 \pm 0.8$ & $0.1 \pm 0.3$ & $0.7 \pm 0.7$ \\
\hline Control subjects & 25 & $8.9 \pm 1.9$ & & & & & & & \\
\hline
\end{tabular}

Note:-RTT indicates Rett syndrome; MZ, monozygotic. Age represents age at time of MR imaging scan. The maximum total clinical severity score is 15 . The "more severe" group included the following mutations: R106W $(n=1), \operatorname{T158M}(n=3), \operatorname{R255X}(n=1), \operatorname{R} 270 X(n=2)$, R294X $(n=2)$, and C-terminal deletions ( $n=3)$. The "less severe" group included the following

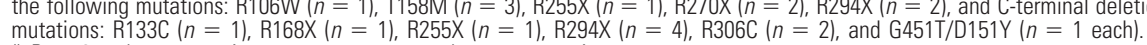

${ }^{*} P<.05$ when comparing more severe versus less severe gait.

umes of the brain, cerebrum, cortical lobes, and sublobar and subcortical regions. The latter was used to provide unbiased confirmation of Talairach-derived results. We also examined the influence of clinical severity on neuroanatomic variation. Based on previous reports, we hypothesized that application of complementary MR imaging techniques would reveal the following: 1) selective reduction of frontal lobe volume in RTT, with a preferential decrease in GM rather than WM;2) greater volumetric reductions in girls with more severe clinical outcomes compared with those with milder clinical presentation; and 3) inverse correlations between regional brain volumes and severity of clinical phenotype.

\section{Methods}

\section{Subjects}

A total of 23 girls with confirmed mutations in the MECP2 gene $(M E C P 2[+]), 22$ fulfilling criteria for RTT, were included. The age range was restricted to $5-12$ years, because this is the period during which mature cortical organization and size are attained. ${ }^{27}$ Twentyfive typically developing girls, used as control subjects, were individually age matched to the RTT girls within 5 months (except for 1 pair of girls who were 7.8 months apart). Typical development in the control group was confirmed by normal full-scale intelligence quotient scores on standardized tests ${ }^{28,29}$ and negative psychiatric diagnoses on a standardized diagnostic interview administered to their mothers (Diagnostic Interview for Children and Adolescents, Revised: Parents' Version). ${ }^{30}$ In addition, all of the control subjects had a negative history of neurologic impairment or neuropsychiatric conditions and showed no clinical evidence of a genetic disorder. Among the $\operatorname{MECP} 2(+)$ girls, there was a previously described pair of monozygotic twins (zygosity confirmed by multiple DNA fingerprinting probes) ${ }^{18}$ carrying a heterozygous 1160 (del26) deletion but different patterns of X chromosome inactivation in peripheral blood (skewed 99:1 versus random 40:60) and discordant clinical presentations. The unaffected twin appeared neurobehaviorally normal, except for minor language delay, which later resolved. The affected twin was included in the MECP2(+) RTT group, whereas the unaffected twin was not included in either the RTT or the control group. MR imaging comparisons between twins were focused on informative regions, as revealed by RTT versus controls and more severe versus less severe RTT phenotype analyses. Further details on the subjects are provided in Table 1.

\section{Clinical Severity Assessment}

Girls with RTT were assessed by one of the authors (S.N.) on 5 parameters of clinical severity, as described previously ${ }^{18}$ : head circum- ference, seizures, respiratory irregularities, scoliosis, and gait abnormalities. Each parameter was scored on a scale from 0 (absent/ normal) to 3 (severe). Table 1 summarizes the clinical characteristics of the RTT cohort.

Although total severity scores (a composite of the above-mentioned 5 parameters) were fairly similar across subjects, we observed pronounced variability in the specific severity profiles of subjects. As described previously, ${ }^{12,16}$ mutations associated with greater severity in one parameter (eg, R294X and head growth deceleration) frequently showed milder severity in other parameters (eg, R294X and scoliosis), and subjects with the same mutation often presented with different severity profiles. To assign meaningful labels of clinical severity to each subject, we focused on the only 2 markedly variable clinical parameters in our cohort: gait abnormalities and scoliosis. In both cases, mild or near-normal presentation (ie, normal walk or no scoliosis; scores of $0-1$ ) was termed "less severe," whereas more affected individuals (ie, scores of 2-3) received the label of "more severe." These 2 parameters did not always yield concordant severity labels; therefore, we examined each categoric scheme separately. Scoliosis severity scores were distributed almost categorically, whereas gait abnormality scores were along a continuum that made them more suitable for clinical-imaging correlational analyses. We have, therefore, presented data and analyses in tables and figures according only to the gait abnormality-based criteria but refer to both criteria in the text.

\section{MR Imaging Acquisition}

Anatomic MR imaging examinations were acquired for all of the subjects on a Signa 1.5T unit (General Electric, Milwaukee, Wis). The imaging protocol included an initial axial T1-weighted scout to ensure proper head positioning, a set of 1.5-mm T1-weighted contiguous coronal MR images, and a spoiled gradient-recalled acquisition sequence (SPGR) (scan parameters: TR, 35-45 ms; TE, 5-7 ms; flip angle, $45^{\circ}$; NEX, 1; matrix size, $256 \times 128 \mathrm{~mm}$; FOV, 20-24 cm). SPGR sequences were reconstructed into $3 \mathrm{D}$ images from these data and resectioned in appropriate planes of interest for analysis.

All of the children were also evaluated with routine brain MR imaging scans, including sagittal $\mathrm{T} 1$ and axial spin-attenuation/T2weighted sequences for the purpose of clinical diagnosis. Various abnormalities, including prominent flow artifacts in the long TR images, mildly prominent ventricles, or increased signal intensity in periatrial deep WM extending into the inferior parietal and occipital regions, were observed in the RTT group. Only 1 clinically abnormal scan was included in the control group; this girl had minimally prominent ventricles and a small pineal cyst, neither of which affected the 


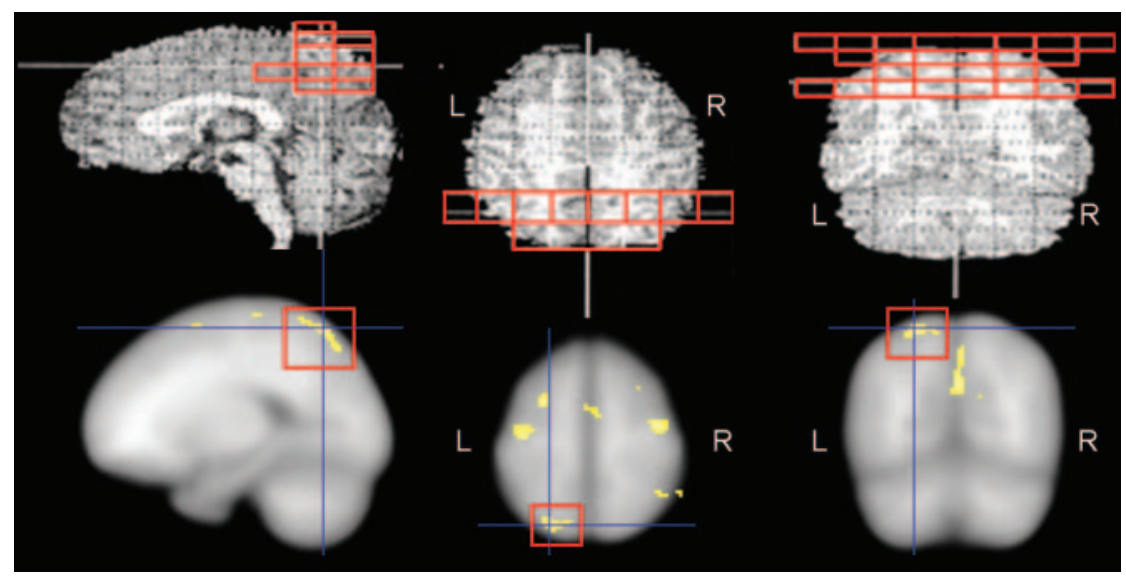

Fig 1. Concordance of Talairach and voxel-based findings in the posterior dorsal parietal lobe in RTT versus control subjects. Talairach-derived data (top row) show preferential bilateral reduction of GM in the posterior dorsal parietal lobe (outlined; only left side shown), corresponding with Brodmann areas 5 and 7. Similarly, VBM-derived data (bottom row) show bilateral GM volume reduction in the posterior dorsal parietal region (outlined), roughly corresponding with Brodmann area 7 (left hemisphere differences are depicted here).

cerebral or cortical measurements included in the analyses. All of the testing protocols were approved by the Johns Hopkins Medicine Institutional Review Board, and informed consent was obtained from all of the parents or legal guardians.

\section{Talairach Data Processing and Analysis}

Using coronal SPGR sequences, raw images were transferred to Macintosh workstations via network connections, and data importation and quantitation were conducted using BrainImage v2.x software. ${ }^{31}$ Images were then filtered using a Gaussian correction algorithm to minimize inhomogeneity, and nonbrain tissue was removed using methods standardized in previous studies. ${ }^{21}$ The "skullstripped" data were resampled into cubic voxels for display in a triplanar view and to allow the placement of a Talairach parcellated grid overlaying each set of images. ${ }^{32-34}$ Three datasets representing proportional pixel maps of GM, WM, and CSF were created by implementation of a multistep semiautomated tissue segmentation algorithm ${ }^{35}$ and introduced into Talairach space for quantitation of region volumes. This approach has been shown to yield high levels of sensitivity and specificity for all of the lobar and sublobar brain regions ${ }^{36}$ and has been validated for use in pediatric study groups, including RTT. ${ }^{33,36}$ All of the measurements were performed by research assistants who were blind to the subjects' diagnoses. Volumetric evaluations included the following CNS compartments: brain, cerebrum (cerebral cortex plus subcortical structures, with the exception of brain stem and cerebellum), brain stem, cerebellum, cerebral cortex (only cortical lobar tissues), 4 cerebral lobes, and corresponding sublobar regions.

\section{Voxel-Based Morphometry Data Analysis}

Processed images corresponding with GM, WM, and CSF pixel maps were analyzed by using Statistical Parametric Mapping software (SPM2). ${ }^{37}$ Before voxel-based morphometry (VBM), raw images were spatially normalized to stereotactic space by using the T1weighted average brain template, and the resultant parameters were applied to GM, WM, and CSF maps. Spatially transformed images were subsequently resampled into $2-\mathrm{mm}^{3}$ isometric voxels and smoothed using a Gaussian kernel of $9 \times 9 \times 9 \mathrm{~mm}^{3}$. Between-group statistical significance was based on a combination of voxel $z$-scores and the spatial distribution of local maxima, after correcting for multiple comparisons across the whole brain volume using a family wise error correction scheme. Voxel clusters that were significantly different between groups were assigned neuroanatomic correlates by using 2 Talairach atlases: the University of Minnesota's Interactive Ta- lairach Atlas ${ }^{38}$ and the University of Texas at San Antonio's Talairach Daemon. ${ }^{39}$ Both atlases were used because of the possibility for small registration discrepancies to lead to different WM voxel regional assignments.

\section{Statistical Analysis}

"Absolute" differences in Talairach-derived volumes were assessed by both analyses of variance and Mann-Whitney $U$ tests without covariates, whereas "relative" volumetric differences were assessed by analyses of covariance, including whole-brain tissue volume as a covariate. Although these data were distributed nonnormally, suggesting nonparametric tests, the latter preclude controlling for the globally reduced brain volume observed in RTT. Therefore, we also created volumetric ratios by dividing compartmental, lobar, and sublobar volumes by whole brain, cerebral, and cortical volumes to differentially examine potential relative/selective changes in smaller compartments. These ratios were analyzed by using nonparametric MannWhitney $U$ tests. Analyses were conducted sequentially, beginning with large compartments and subsequently examining smaller volumes. When necessary, analyses were Bonferroni corrected for multiple comparisons, with significance defined as a postcorrection $P$ value $\leq .05$ and trends defined as $.05 \leq P<.1$. As mentioned above, GM and WM voxel intensities were analyzed by the SPM2, as in previous morphometric studies of a variety of neurologic disorders. ${ }^{40-42}$

The above-mentioned analyses were conducted in the following comparative groups: $M E C P 2(+)$ RTT subjects versus control subjects and girls with less severe versus more severe clinical presentations, using both the gait abnormality- and scoliosis-based severity criteria. In addition, we used descriptive statistics to examine brain regions in the pair of monozygotic twins with discordant clinical presentations.

\section{Results}

\section{MECP2(+) RTT Subjects versus Control Subjects}

As shown in On-line Table 1, significant reductions in whole brain tissue, as well as in the absolute volumes of cerebral, cortical, and subcortical tissue, were observed in girls with confirmed MECP2 mutations. However, these regions showed no relative volumetric reductions when controlling for whole brain tissue volume. Significant absolute reductions in whole brain GM and WM were observed, as well as absolute and relative reductions in cerebral GM and WM $(P<.1)$ and cortical WM $(P<.04)$. These differences were noted bilaterally. Significant absolute and relative volumetric reductions were 


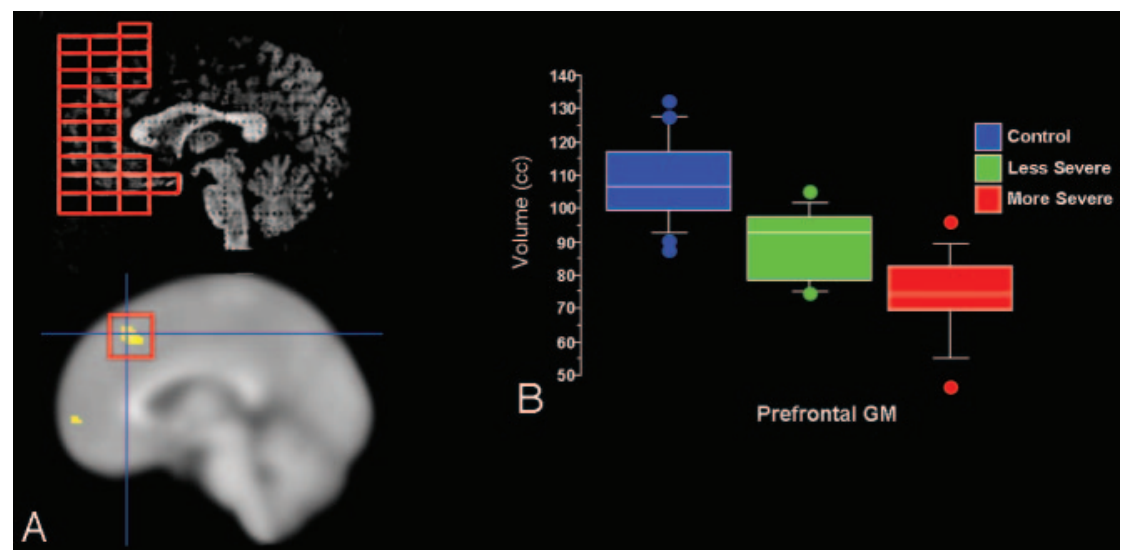

Fig 2. Concordance of Talairach and voxel-based findings in the prefrontal GM in more severe versus less severe RTT subjects. Girls with less severe clinical presentation, according to gait abnormality criterion, showed relative preservation of prefrontal GM volumes, corresponding roughly with Brodmann areas 10/46. A, Top left depicts Talairach-derived data, whereas bottom left shows VBM-derived data. $B$, Boxplots (percentiles) of Talairach-based prefrontal GM volumes illustrate that girls with less severe clinical presentation show volumes intermediate between control subjects and those with more severe phenotype.

noted in GM of the parietal lobe $(P<.05)$, predominantly in the left $(P<.03)$ but not the right $(P<.16)$ hemisphere. We also observed trend-level reductions in WM volumes in the temporal lobe in the right hemisphere $(P<.1)$. Subparietal GM reductions were observed in the left inferior parietal lobule $(P<.06)$ and bilateral posterior dorsal parietal region $(P<$ .001 ; Fig 1 and On-line Table 2).

VBM showed only differences in GM comparing RTT and control subjects. The areas of the most significant GM reduction in RTT subjects were the right cingulate and middle occipital gyri (Brodmann areas 32 and 19/39, respectively), bilateral posterior dorsal parietal lobe (Brodmann area 7), left middle frontal gyrus (Brodmann area 10), and bilateral precentral and postcentral gyri (Brodmann areas $4 / 6$ and 5/7, respectively; Fig 1).

\section{Less Severe versus More Severe Clinical Presentation}

Using the gait abnormality-based criteria, girls with more and less severe clinical presentation did not differ significantly in total brain tissue volume, though less severely affected girls had significantly larger brain GM volumes, which were driven by relative sparing of frontal and temporal GM. Although parietal GM volumes were slightly larger in less affected girls, GM volumes in the occipital lobe were not significantly different from those in girls with more severe clinical outcomes (Online Table 1). Within the frontal lobe, prefrontal GM volume was relatively larger bilaterally in girls with milder clinical severity, whereas in the temporal lobe milder clinical severity was associated with relative preservation of the anterior superior temporal gyrus GM (On-line Table 2 and Fig 2).

Using the scoliosis-based criteria, we observed smaller total brain tissue volumes in girls with greater clinical severity, which was driven by significant GM reductions in all 4 of the cerebral lobes (though to a lesser extent occipitally). In correspondence with the Talairach-based findings, uncorrected VBM results suggested greater preservation of anterior frontal and temporal GM in less severely affected RTT subjects.

\section{Analysis of Monozygotic Twins}

Descriptive analyses of brain volumes in the monozygotic twins relative to one another and to control subjects revealed that the unaffected twin had total brain and lobar volumes within the range seen in control subjects. By contrast, the affected twin showed reduced volumes, particularly with respect to GM, in the whole brain and frontal, parietal, and temporal lobes. However, both twins showed occipital GM volumes analogous to those observed in control subjects (Fig 3).

\section{Effect of Age on Imaging Parameters}

We did not observe a significant relationship between age and volume of any brain region in RTT or control subjects, nor did we observe significant differences in age-brain relationships between girls with RTT and control subjects. Only under the scoliosis-based criteria were more severely affected girls significantly older than less affected RTT subjects. Nonetheless, volumetric findings remained significant after the introduction of age as a covariate in parametric models.

\section{Clinical-Imaging Correlations}

Comparing regional volumes found to be different in the aforementioned comparisons with measures of clinical severity, we observed that, in general, volumetric reductions were associated with higher total clinical severity scores. When examining specific subparietal regions, we observed that posterior dorsal parietal GM volumes were negatively correlated with total severity scores $(P<.04)$, but not any specific clinical measure, whereas inferior parietal lobule GM volumes were negatively correlated with both total severity scores $(P<.01)$ and severity of gait abnormalities $(P<.02)$. Within the frontal lobe, both prefrontal and premotor GM volumes were negatively correlated with total $(P<.01)$ and gait abnormality $(P<.01)$ severity scores.

\section{Discussion}

This study used complementary MR imaging approaches to provide evidence for a selective volumetric reduction of parietal lobe GM in girls with RTT, beyond the known global decrease in brain volume. Contrary to our initial hypotheses, these reductions were localized consistently in the dorsal parietal region, regardless of mutation or clinical severity. Comparison of volumetric profiles of monozygotic twins discordant for the RTT phenotype emphasized relative occipital lobe preservation in this disorder. Although relative reduction of parietal GM may be understood as a basic neuroanatomic feature of RTT, greater reduction of the anterior frontal lobe appears to be a correlate of severity in the RTT phenotype as revealed by comparisons between more and less affected groups. WM reductions were also present but were rather mild and relatively diffuse. Also in line with our hypotheses, GM volumes in parietal and frontal regions correlated negatively 


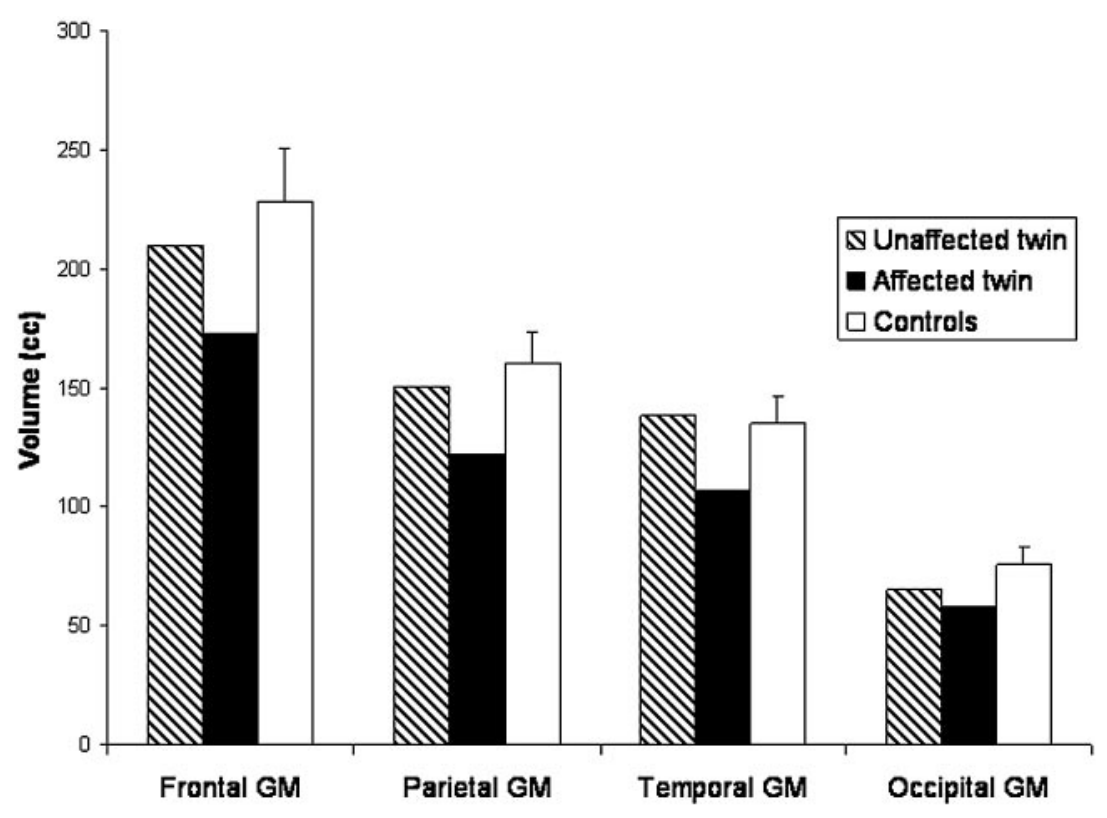

with measures of clinical severity, most notably gait abnormalities and overall clinical severity.

Our results confirm previous findings of reduced brain volume in RTT, namely global brain hypoplasia, ${ }^{43,44}$ preferential involvement of $\mathrm{GM},{ }^{22,24}$ and no evidence for progressive reduction of brain tissue. ${ }^{22,24}$ However, in contrast to earlier reports implicating predominant involvement of prefrontal, posterior frontal, and anterior temporal regions, ${ }^{22,24}$ our data suggest that reductions in parietal GM are the primary neuroanatomic feature of RTT, with preferential decreases in anterior frontal cortex as a secondary marker of increasing clinical severity. This discordance may be explained by 3 main possibilities. First, the present study focused on girls with confirmed MECP2 mutations, whereas earlier studies tended to include only girls with the "classic" RTT phenotype, regardless of their mutation status, which may have biased their samples to subjects with more severe clinical presentations and greater frontal and temporal involvement. Second, contrary to the measurement protocols used in previous studies, we used the pediatric adaptation of the Talairach semiautomated system, a protocol that has been validated for patients with RTT and other neurodevelopmental disorders. ${ }^{33,36}$ Third, whereas most neuroimaging studies have used a single analytical approach, here we applied complementary unbiased and directed methods. The present study also included a comparison between monozygotic twins discordant for the RTT phenotype, which confirmed our previous study demonstrating greater cortical involvement in the affected $\operatorname{twin}^{24}$ and the relative sparing of the occipital cortex in RTT reported in several neuroanatomic and neuroimaging studies. ${ }^{22-24,45,46}$ Neurobiologically, the preferential volumetric reductions in dorsal parietal GM found in this study support the hypothesis of early dendritic growth arrest in RTT, ${ }^{20,27,47-49}$ because the most affected cortical regions are among the first to develop. ${ }^{27,48}$ On the other hand, the mild and diffuse reductions in cortical WM are most likely related to axonal pathology (ie, decreased axonal diameter and/or branching) and not to myelination abnormalities, because no changes in $\mathrm{T} 2$ signal intensity or
Fig 3. Cerebral lobar GM volumes in monozygotic twins discordant for the RTT phenotype. Note that the unaffected twin showed frontal, parietal, and temporal GM volumes within the normal control range (depicted as mean $+1 \mathrm{SD}$ ), whereas the affected twin had smaller volumes in these regions, with the exception of the occipital GM volume which was at nearly identical levels in both twins and only slightly below the normal control range.

other related parameters have been observed or reported in the literature. ${ }^{50,51}$

From a functional standpoint, parietal GM reductions, particularly in the dorsal parietal region and inferior parietal lobule, correspond with known deficits in RTT and generally accord with the imaging-clinical correlations observed in the present study. Although considerable emphasis has been placed on the association between frontal dysfunction and cognitive and motor deficits, parietal reductions are consistent with deficits in sequential movements and tactile information processing (including pain) that typify RTT. ${ }^{14,15}$ The role of the posterior dorsal parietal cortex in the regulation of ocular and limb movement via connections with the premotor cortex is relatively well established, ${ }^{52,53}$ and the adjacent inferior parietal lobule is implicated in tactile processing and sensorimotor integration, ${ }^{54}$ including hand proprioception for functional object use. ${ }^{55}$ Selective anterior frontal reductions in girls with more severe phenotypes may underlie greater cognitive and motor involvement in these individuals.

The time course of these abnormalities remains to be determined. Although most girls with RTT do not show major neurology delay or impairment during the first year of life, several studies have demonstrated abnormal movements and other neurobehavioral differences ${ }^{56,57}$ and deceleration of head growth in a significant proportion of RTT patients during the second half of the first year. ${ }^{20}$ Therefore, future MR imaging studies should focus on younger subjects, possibly within the first year of life, to determine whether selective parietal abnormalities are present at this stage, even before reductions in head circumference become severe. ${ }^{3,13,14}$ Careful manual delineation of the posterior dorsal parietal lobe, along with complementary analyses of cortical thickness and gyration, may clarify the nature of changes in this region. MR spectroscopy studies, which have already provided some evidence for metabolic impairment in the parietal and frontal lobes in RTT, ${ }^{58}$ also may shed some light on the cellular substrates of the neuroanatomic abnormalities reported here. It also may be elucidative to re-examine animal models of RTT to determine using similar methods whether selective cortical changes are present, though the difficulties of correlating murine and human neuroanatomy and issues of partial versus complete lack of Mecp2 expression in the mouse remain problematic. ${ }^{59}$ Lastly, the data presented here open the possibility of establishing neuroanatomic profiles for each MECP2 mutation, though large cohorts will undoubtedly be required. 


\section{Conclusion}

This study provides evidence that, despite the predicted generalized and pronounced cortical GM involvement in RTT, there is a distinctive pattern affecting the dorsal parietal region, as demonstrated by 2 complementary MR imaging approaches. Beyond this, greater anterior frontal involvement is related to greater clinical severity, in accordance with our initial hypotheses. With further delineation of selective neuroanatomic deficits, MR imaging morphometry may eventually complement existing clinical classification of RTT and determine regions of vulnerability that respond to definitive treatments.

\section{Acknowledgments}

We thank L. Uribe, M. Abrams, P. Mazur-Hopkins, and M. Lager for assistance in data processing and analysis.

\section{References}

1. Rett A. Über eine eiggenartiges hirnatrophisches Syndrome bei hyperammonamie im Kindesalter. Wien Med Wochenschr 1966;116:723-28

2. Naidu S, Murphy M, Moser HW, et al. Rett syndrome: natural history in 70 cases. Am J Med Genet 1986;24:61-72

3. Naidu S, Hyman S, Harris EL, et al. Rett syndrome studies of natural history and search for a genetic marker. Neuropediatrics 1995;26:63-66

4. Naidu S. Rett syndrome: a disorder affecting early brain growth. Ann Neurol 1997;42:3-10

5. Amir RE, Van den Veyver IB, Wan M, et al. Rett syndrome is caused by mutations in X-linked MECP2, encoding methyl-CpG-binding protein 2. Nat Genet 1999;23:185-88

6. Bienvenu T, Carrie A, deRoux N, et al. MECP2 mutations account for most cases of typical forms of Rett syndrome. Hum Mol Genet 2000;9:1377-84

7. Huppke P, Laccone F, Kramer N, et al. Rett syndrome: analysis of MECP2 and clinical characterization of 31 patients. Hum Mol Genet 2000;9:1369-75

8. Van den Veyver IB, Zoghbi H. Methyl-CpG-binding protein 2 mutations in Rett syndrome. Curr Opin Genet Dev 2000;10:275-79

9. Xiang F, Buervenich S, Nicolao P, et al. Mutation screening in Rett syndrome patients. J Med Genet 2000;37:250-55

10. Christodoulou J, Grimm A, Maher T, et al. RettBASE: the IRSA MECP2 variation database - a new mutation database in evolution. Hum Mutat 2003;21:466-72

11. Mount RH, Hastings RP, Reilly S, et al. Towards a behavioral phenotype for Rett syndrome. Am J Ment Retard 2003;108:1-12

12. Naidu S, Bibat G, Kratz L, et al. Clinical variability in Rett syndrome. J Child Neurol 2003;18:662-68

13. Hagberg B, Witt-Engerstrom I. Rett syndrome: a suggested staging system for describing impairment profile with increasing age towards adolescence. $\mathrm{Am} \mathrm{J}$ Med Genet 1986;24:47-59

14. Trevathan E, Moser H, for the Rett Syndrome Diagnostic Criteria Work Group. Diagnostic criteria for Rett syndrome. The Rett Syndrome Diagnostic Criteria Work Group. Ann Neurol 1988;23:425-28

15. Hagberg B. Clinical delineation of Rett syndrome variants. Neuropediatrics 1995;26:62

16. Colvin L, Leonard H, de Klerk N, et al. Refining the phenotype of common mutations in Rett syndrome. J Med Genet 2004;41:25-30

17. Robertson L, Hall SE, Jacoby P, et al. The association between behavior and genotype in Rett syndrome using the Australian Rett Syndrome Database. Am J Med Genet 2006;141B:177-83

18. Hoffbuhr K, Devaney JM, LaFleur B, et al. MECP2 mutations in children with and without the phenotype of Rett syndrome. Neurology 2001;56:1486-95

19. Huppke P, Held M, Hanefeld F, et al. Influence of mutation type and location on phenotype in 123 patients with Rett syndrome. Neuropediatrics 2002;33:63-68

20. Kaufmann WE, Pearlson GD, Naidu S. The neuroanatomy of Rett syndrome: neuropathological and neuroimaging studies. Rivista Medica 1998;4:189-200

21. Abrams MT, Mazur-Hopkins P, Pearlson GD, et al. Brain MRI changes in Rett syndrome: cross-sectional and serial data. Soc Neurosci 1999;25:488a.

22. Reiss AL, Faruque F, Naidu S, et al. Neuroanatomy of Rett syndrome: a volumetric imaging study. Ann Neurol 1993;34:227-34

23. Armstrong D, Dunn JK, Antalffy B, et al. Selective dendritic alterations in the cortex of Rett syndrome. J Neuropathol Exp Neurol 1995;54:195-201

24. Subramaniam B, Naidu S, Reiss AL. Neuroanatomy in Rett syndrome: cerebral cortex and posterior fossa. Neurology 1997;48:399-407
25. Yoshikawa H, Fueki N, Suzuki H, et al. Cerebral blood flow and oxygen metabolism in the Rett syndrome. Brain Dev 1992;14(suppl):S69-74

26. Bjure J, Uvebrant P, Vestergren E, et al. Regional cerebral blood flow abnormalities in Rett syndrome. Eur Child Adolesc Psychiatry 1997;6(suppl):64-66

27. Kaufmann WE. Cortical histogenesis. In: Aminoff MJ, Daroff RB, eds. Encyclopedia of the Neurological Sciences. San Diego: Academic Press; 2003:Vol. 1;777-84

28. Wechsler DL. The Wechsler Intelligence Scale for Children-Revised. San Antonio The Psychological Corporation; 1974

29. Wechsler DL. The Wechsler Intelligence Scale for Children - III. San Antonio: The Psychological Corporation; 1991

30. Reich MJ, Shayka T, Taibleson C. The Diagnostic Interview for Children and Adolescents-Revised. St Louis: Washington University Press; 1991

31. Behavioral Neurogenetics and Neuroimaging Research Center. BrainImage ( $v$ 2.x). Baltimore: Kennedy Krieger Institute; 1997

32. Andreasen NC, Rajarethinam R, Cizadlo T, et al. Automatic atlas-based volume estimation of human brain regions from MR images. J Comp Assist 1996;20:98-106

33. Kaplan DM, Liu AM, Abrams MT, et al. Application of an automatic parcellation method to the analysis of pediatric brain volumes. Psychiatry Res 1997;76:15-27

34. Talairach J, Tournoux P, eds. Co-Planar Stereotaxic Atlas of the Human Brain. New York: Thieme Medical Publishers, Inc; 1988

35. Otsu N. A threshold selection method from gray-level histograms. IEEE Trans Syst Man Cybern 1979;9:62-66

36. Kates WR, Warsofsky IS, Patwardhan A, et al. Automated Talairach atlas-based parcellation and measurement of cerebral lobes in children. Psychiatry Res 1999;91:11-30

37. Statistical Parametric Mapping (SPM2). London: Wellcome Department of Imaging Neuroscience; 2003

38. NeuroVia Talairach Atlas. Minneapolis-St Paul: University of Minnesota; 2007

39. Research Imaging Center. Talairach Daemon Client (v. 2). San Antonio: University of Texas Health Science Center; 2005

40. White NS, Alkire MT, Haier RJ. A voxel-based morphometric study of nondemented adults with Down syndrome. Neuroimage 2003;20:393-403

41. Boddaert N, Chabane N, Gervais H, et al. Superior temporal sulcus anatomical abnormalities in childhood autism: a voxel-based morphometry MRI study. Neuroimage 2004;23:364-69

42. Boddaert N, Mochel F, Meresse I, et al. Parieto-occipital grey matter abnormalities in children with Williams syndrome. Neuroimage 2006;30:721-25

43. Casanova MF, Naidu S, Goldberg T, et al. Quantitative magnetic resonance imaging in Rett syndrome. J Neuropsychiatry Clin Neurosci 1991;3:66-72

44. Murakami JW, Courchesne E, Haas RH, et al. Cerebellar and cerebral abnormalities in Rett syndrome: a quantitative MR analysis. AJR Am J Roentgenol 1992;159:177-83

45. Kaufmann WE, MacDonald SM, Altamura CR. Dendritic cytoskeletal protein expression in mental retardation: an immunohistochemical study of the neocortex in Rett syndrome. Cereb Cortex 2000;10:992-1004

46. Kaufmann WE, Worley PF, Taylor CV, et al. Cyclooxygenase-2 expression during rat neocortical development and in Rett syndrome. Brain Dev 1997;19:25-34

47. Armstrong DD, Dunn K, Antalffy B. Decreased dendritic branching in frontal, motor and limbic cortex in Rett syndrome compared with trisomy 21. J Neuropathol Exp Neurol 1998;57:1013-17

48. Kaufmann WE, Naidu S, Budden S. Abnormal expression of microtubuleassociated protein 2 (MAP-2) in neocortex in Rett syndrome. Neuropediatrics 1995;26:109-13

49. Armstrong DD. Neuropathology of Rett syndrome. Ment Retard Dev Disabil Res Rev 2002;8:72-76

50. Horska A, Naidu S, Herskovits EH, et al. Quantitative 1H MR spectroscopic imaging in early Rett syndrome. Neurology 2000;54:715-22

51. Gokcay A, Kitis O, Ekmekci, et al. Proton MR spectroscopy in Rett syndrome. Comput Med Imaging Graph 2002;26:271-75

52. Buneo CA, Andersen RA. The posterior parietal cortex: sensorimotor interface for the planning and online control of visually guided movements. $\mathrm{Neu}$ ropsychologia 2006;44:2594-606

53. Fogassi L, Luppino G. Motor functions of the parietal lobe. Curr Opin Neurobiol 2005;15:626-31

54. Hillis AE, Chang S, Heidler-Gary J, et al. Neural correlates of modality-specific spatial extinction. J Cogn Neurosci 2006;18:1889-98

55. Buxbaum LJ, Kyle KM, Tang K, et al. Neural substrates of knowledge of hand postures for object grasping and functional object use: evidence from fMRI. Brain Res 2006;1117:175-85

56. Einspieler C, Kerr AM, Prechtl HF. Is the early development of girls with Ret disorder really normal? Pediatr Res 2005;57:696-700

57. Einspieler C, Kerr AM, Prechtl HF. Abnormal general movements in girls with Rett disorder: the first four months of life. Brain Dev 2005;27(suppl 1):S8-13

58. Hoffbuhr KC, Moses LM, Jerdonek, et al. Associations between MECP2 mutations, X-chromosome inactivation, and phenotype. Ment Retard Dev Disabil Res Rev 2002;8:99-105

59. Stearns NA, Schaevitz LR, Bowling $\mathrm{H}$, et al. Behavioral and anatomic abnormalities in Mecp2 mutant mice: a model for Rett syndrome. Neuroscience 2007; 146:907-21 